Contents List available at RAZI Publishing

Matriks Sains Matematik (MSMK)

Journal Homepage: http://www.razipublishing.com/journals/matriks-sains-matematik

https://doi.org/10.26480/msmk.02.2017.22.26

\title{
INTUITIONISTIC FUZZY INTERIOR IDEAL OF SEMIGROUP BASED ON INTUITIONISTIC FUZZY POINT

Hidayat Ullah Khan ${ }^{*}$, Nor Haniza Sarmin ${ }^{2}$, Asghar Khan ${ }^{3}$, and Faiz Muhammad Khan ${ }^{4}$ and Muhammad Taj

\author{
${ }^{1}$ Department of Mathematics, University of Malakand, Khyber Pakhtunkhwa, Pakistan \\ ${ }^{2}$ Department of Mathematical Sciences, Faculty of Science Universiti Teknologi Malaysia 81310 UTM, Johor Bahru, Johor, Malaysia \\ ${ }^{3}$ Department of Mathematics, Abdul Wali Khan University Mardan, Khyber Pakhtunkhwa, Pakistan \\ ${ }^{4}$ Department of Mathematics and Statistics, University of Swat, Khyber Pakhtunkhwa, Pakistan \\ ${ }_{5}^{5}$ Department of Mathematics, University of Azad Jammu and Kashmir Muzaffarabad 13100, Azad Kashmir, Pakistan \\ *Corresponding Author email: hidayatullak@yahoo.com
}

This is an open access article distributed under the Creative Commons Attribution License, which permits unrestricted use, distribution, and reproduction in any medium, provided the original work is properly cited

\section{ARTICLE DETAILS}

\section{Article History:}

Received 7 July 2017

Accepted 11 October 2017

Available online 6 November 2017

Keywords:

Intuitionistic fuzzy interior ideal; $(\bar{\epsilon}, \bar{\epsilon} \vee \bar{q})$-fuzzy interior ideal;

$(\bar{\epsilon}, \bar{\epsilon})$-fuzzy interior ideal.

2000 Mathematics Subject

Classification: 06F05; 20M12;

$08 \mathrm{~A} 72$.

\section{ABSTRACT}

The intuitionistic fuzzification of the notion of an interior ideal in ordered semigroups is considered. The purpose of this study is to introduce the notion of $(\bar{\epsilon}, \bar{\epsilon} \vee \bar{q})$-intuitionistic fuzzy interior ideals and $(\bar{\epsilon}, \bar{\epsilon})$-intuitionistic fuzzy interior ideals of semigroups. The important milestone of the present paper is to link ordinary intuitionistic fuzzy interior ideals, $(\bar{\epsilon}, \bar{\epsilon})$-intuitionistic fuzzy interior ideals and $(\bar{\epsilon}, \bar{\epsilon} \vee \bar{q})$-intuitionistic fuzzy interior ideals. Moreover, semigroups are characterized by the properties of these new notions.

\section{INTRODUCTION}

Fuzzy set theory introduced by Zadeh is a useful tool to describe situations in which the data are imprecise or vague and handle such situations by attributing a degree to which a certain object belongs to a set [1]. Just after the establishment of fuzzy set theory, it has developed in several directions and is discovering applications in a wide variety of fields. Rosenfeld used this idea to develop the theory of fuzzy groups [2]. Fuzzy set theory provides a natural framework for generalization of the basic notions of algebra. But there is no means to incorporate the hesitation or uncertainty in the membership degrees. Atanassov introduced the concept of intuitionistic fuzzy sets in 1986, which constitute an extension of a fuzzy set theory [3]. Since then, many researchers have investigated this topic such as intuitionistic fuzzy group [4-6]. Intuitionistic fuzzy sets give both a membership degree and a non-membership degree, where sum of membership degree and non-membership degree needs to be less or equal to 1 . $\mathrm{Pu}$ and Liu introduced the notions of "belongings" and "quasicoincidence" of fuzzy point and fuzzy set [7]. Bhakat and Das introduced $(\in, \in \vee q)$-fuzzy subgroup as a generalization of Rosenfeld's fuzzy subgroups by using the combine notions of "belongings $(\in)$ " and "quasi-coincidence $(q)$ " of fuzzy point and fuzzy set [8]. In semigroup Jun and Song gave the concept of an $(\alpha, \beta)$-fuzzy interior ideal, which is a generalization of a fuzzy interior ideal by using the idea of quasi-coincidence of a fuzzy point with a fuzzy set [9]. In Davvaz and Khan discussed some characterizations of regular ordered semigroups in terms of $(\alpha, \beta)$-fuzzy generalized bi-ideals,

where $\alpha, \beta \in\{\in, q, \in \vee q, \in \wedge q\}$ and $\alpha \neq \in \wedge q$ [10]. Some authors have investigated similar type of generalizations of other algebraic structures. Kazanci and Yamak introduced the concept of a generalized fuzzy bi-ideal in semigroups and gave some properties of fuzzy bi-ideals in terms of $(\in, \in \vee q)$-fuzzy bi-ideals [11]. A group of scientists characterized regular semigroups by the properties of $(\in, \in \vee q)$-fuzzy ideals, bi-ideals and quasi-ideals [12]. In a research, author has introduced more generalized forms of $(\alpha, \beta)$-fuzzy ideals and defined $\left(\in, \in \vee q_{k}\right)$-fuzzy ideals of semigroups [13]. Kazanci and Yamak [11] gave $(\bar{\epsilon}, \bar{\epsilon} \vee \bar{q})$-fuzzy biideals of a semigroup. Another researcher introduced $(\in, \in \vee q)$-fuzzy interior ideals of ordered semigroups and investigated some characterizations of ordered semigroups in terms of $(\in, \in \vee q)$-fuzzy interior ideals [14]. The notion of $(\bar{\epsilon}, \bar{\epsilon} \vee \bar{q})$-fuzzy interior ideal of semigroup is introduced in [15]. A studied of $\left(\bar{\epsilon}, \bar{\epsilon} \vee \bar{q}_{k}\right)$-fuzzy ideals, generalized bi-ideals and quasi-ideals of a semigroup and characterized regular semigroups by the properties of these ideals [16].

Kim and Jun introduced the notion of an intuitionistic fuzzy interior ideal of a semigroup $S$ and gave its characterization [5]. In a study conducted by some researcher redefined $(\alpha, \beta)$-intuitionistic fuzzy subgroups [6]. The readers are referred to for further reading regarding $(\alpha, \beta)$-fuzzy subsets and their generalization [17-24].

In this paper, we introduce the notion of $(\bar{\epsilon}, \bar{\epsilon} \vee \bar{q})$-intuitionistic fuzzy interior ideals of a semigroup and discuss some interesting results and investigate the relationships among ordinary intuitionistic fuzzy interior ideals, $(\bar{\epsilon}, \bar{\epsilon})$-intuitionistic fuzzy interior ideals and $(\bar{\epsilon}, \bar{\epsilon} \vee \bar{q})$ intuitionistic fuzzy interior ideals of semigroups.

\section{BASIC DEFINITIONS AND PRELIMINARIES}

In what follows $S$ will represent a semigroup unless otherwise stated. A non-empty subset $A$ of $S$ is called a subsemigroup of $S$ if $A^{2} \subseteq A$. 
A non-empty subset $A$ of a semigroup $S$ is called an interior ideal of $S$ if:

$$
\begin{aligned}
\text { i. } & A^{2} \subseteq A, \\
\text { ii. } & S A S \subseteq A .
\end{aligned}
$$

An intuitionistic fuzzy subset (briefly IFS) $A$ in a non-empty set $X$ is an object having the form $A=\left\{\left\langle x, \mu_{A}(x), \gamma_{A}(x)\right\rangle \mid x \in X\right\}$, where the function $\mu_{A}: X \rightarrow[0,1]$ and $\gamma_{A}: X \rightarrow[0,1]$ denote the degree of membership (namely $\mu_{A}(x)$ ) and the degree of non-membership (namely $\gamma_{A}(x)$ ) of each element $x \in X$ to the set $A$, respectively and $0 \leq \mu_{A}(x)+\gamma_{A}(x) \leq 1$ for all $x \in X$. For the sack of simplicity, we shall use the symbol $A=\left\langle x, \mu_{A}, \gamma_{A}\right\rangle$ for the intuitionistic fuzzy set $A=\left\{\left\langle x, \mu_{A}(x), \gamma_{A}(x)\right\rangle \mid x \in X\right\}$. Let $A=\left\langle x, \mu_{A}, \gamma_{A}\right\rangle$ be an IFS of $S$, then $A$ is called an intuitionistic fuzzy subsemigroup of $S$ if for all $x, y \in S$

$$
\left(\mu_{A}(x y) \geq \min \left\{\mu_{A}(x), \mu_{A}(y)\right\}\right.
$$

And

$$
\gamma_{A}(x y) \leq \max \left\{\gamma_{A}(x), \gamma_{A}(y)\right\}
$$

\section{$2.1 \quad$ Definition}

Let $A=\left\langle x, \mu_{A}, \gamma_{A}\right\rangle$ be an intuitionistic fuzzy subset of a semigroup $S$. Then $A$ is called an intuitionistic fuzzy interior ideal of $S$ if for all $x, y, a \in S$ the following conditions hold;

( $\left.C_{1}\right) \mu_{A}(x y) \geq \min \left\{\mu_{A}(x), \mu_{A}(y)\right\}$ and $\gamma_{A}(x y) \leq \max \left\{\gamma_{A}(x), \gamma_{A}(y)\right\}$.

$\left(C_{2}\right) \mu_{A}(x a y) \geq \mu_{A}(a)$ and $\gamma_{A}(x a y) \leq \gamma_{A}(a)$.

The $\mu_{A}$-level cut and $\gamma_{A}$-level cut of an intuitionistic fuzzy subset $A=\left\langle x, \mu_{A}, \gamma_{A}\right\rangle$ of a semigroup $S$ respectively are denoted and defined as $U\left(\mu_{A} ; t\right)=\left\{x \in S \mid \mu_{A}(x) \geq t\right\} \quad$ and $\quad L\left(\gamma_{A} ; s\right)=\left\{x \in S \mid \gamma_{A}(x) \leq s\right\}$, where $t \in(0,1]$ and $s \in[0,1)$.

The $\left(\mu_{A}, \gamma_{A}\right)$-level $(t, s)$-cut is defined as

$$
C_{(t, s)}(A)=\left\{x \in S \mid \mu_{A}(x) \geq t \text { and } \gamma_{A}(x) \leq s\right\}
$$

It is clear that $C_{(t, s)}(A)=U\left(\mu_{A} ; t\right) \cup L\left(\gamma_{A} ; s\right)$.

\section{$2.2 \quad$ Theorem [3]}

Let $A=\left\langle x, \mu_{A}, \gamma_{A}\right\rangle$ be an intuitionistic fuzzy subset of $S$. Then $A=\left\langle x, \mu_{A}, \gamma_{A}\right\rangle$ is an intuitionistic fuzzy interior ideal of $S$ it and only if $(\forall t \in(0,1], s \in[0,1)) C_{(t, s)}(A)(\neq \phi)$ is an interior ideal of $S$.

\section{$2.3 \quad$ Definition}

Let $x$ be a point of a non-empty set $X$. If $t \in(0,1], s \in[0,1)$ are two real numbers such that $0<t+s \leq 1$, then the IFS of the form $[x ;(t, s)]=\left[x ; x_{t}, 1-x_{1-s}\right]$ is called an intuitionistic fuzzy point (IFP for short) in $X$, where $t$ (resp. $s$ ) is the degree of membership (resp. nonmembership) of $[x ;(t, s)]$ and $x \in X$ is the support of $[x ;(t, s)]$.

\section{4}

\section{Definition}

Consider an IFP $[x ;(t, s)]$ in $S$, an IFS $A=\left\langle x, \mu_{A}, \gamma_{A}\right\rangle$ and $\alpha \in\{\epsilon, q, \in \vee q\}$, we define $[x ;(t, s)] \alpha A$ as follows;

(i). By $[x ;(t, s)] \in A$ (resp. $[x ;(t, s)] q A)$ we mean $\mu_{A}(x) \geq t$ and $\gamma_{A} \leq s$ (resp. $\mu_{A}(x)+t>1$ and $\gamma_{A}+s<1$ ) and say that $[x ;(t, s)]$ belongs to (resp. quasi-coincident with) an IFS $A=\left\langle x, \mu_{A}, \gamma_{A}\right\rangle$.

(ii). $[x ;(t, s)] \in \vee q A \quad$ (resp. $[x ;(t, s)] \in \wedge q A$ ) means that $[x ;(t, s)] \in A$ or $[x ;(t, s)] q A$ (resp. $[x ;(t, s)] \in A$ and $[x ;(t, s)] q A$ ). (iii). By $[x ;(t, s)] \overline{\alpha A}$ we mean that $[x ;(t, s)] \alpha A$ do not hold.

\section{$2.5 \quad$ Definition}

For a non-empty subset $A$ of $S$ the characteristic function $\chi_{A}=<x, \mu_{\chi_{A}}(x), \gamma_{\chi_{A}}(x)>$ of $A$ is defined by

$$
\begin{aligned}
& \mu_{\chi_{A}}(x): S \rightarrow[0,1], x \mapsto \mu_{\chi_{A}}(x):= \begin{cases}1 & \text { if } x \in A, \\
0 & \text { if } x \notin A,\end{cases} \\
& \gamma_{\chi_{A}}(x): S \rightarrow[0,1], x \mapsto \gamma_{\chi_{A}}(x):= \begin{cases}0 & \text { if } x \in A, \\
1 & \text { if } x \notin A .\end{cases}
\end{aligned}
$$

\subsection{Proposition}

A non-empty subset $A$ of $S$ is an intuitionistic fuzzy interior ideal of $S$ if and only if the characteristic function $\chi_{A}=<x, \mu_{\chi_{A}}(x), \gamma_{\chi_{A}}(x)>$ of $A$ is an intuitionistic fuzzy interior ideal of $S$.

\section{3. $(\bar{\epsilon}, \bar{\epsilon} \vee \bar{q})$-INTUITIONISTIC FUZZY INTERIOR IDEALS}

In this section, we introduce $(\bar{\epsilon}, \bar{\epsilon} \vee \bar{q})$-intuitionistic fuzzy interior ideals of a semigroup $S$.

\subsection{Definition}

An intuitionistic fuzzy subset $A=<x, \mu_{A}(x), \gamma_{A}(x)>$ of $S$ is called an $(\bar{\epsilon}, \bar{\epsilon} \vee \bar{q})$-intuitionistic fuzzy interior ideal of $S$, if for all $x, y, a \in S$, $t, t_{1}, t_{2} \in(0,1]$ and $s, s_{1}, s_{2} \in[0,1)$, satisfies the following conditions:

$\left(C_{3}\right) . \quad\left[x y ; \min \left\{t_{1}, t_{2}\right\}, \max \left\{s_{1}, s_{2}\right\}\right] \bar{\epsilon} A \rightarrow\left[x ;\left(t_{1}, s_{1}\right)\right] \bar{\epsilon} \vee \bar{q} A \quad$ or $\left[y ;\left(t_{2}, s_{2}\right)\right] \bar{\epsilon} \vee \bar{q} A$,

$\left(C_{4}\right) . \quad[x a y ;(t, s)] \bar{\epsilon} A \rightarrow[a ;(t, s)] \bar{\epsilon} \vee \bar{q} A$.

\section{$3.2 \quad$ Example}

Consider a semigroup $S=\{a, b, c, d, e\}$ with the following multiplication table:

Table 1: Multiplication of $S=\{a, b, c, d, e\}$

\begin{tabular}{|l|l|l|l|l|l|}
\hline$\cdot$ & $a$ & $b$ & $c$ & $d$ & $e$ \\
\hline$a$ & $a$ & $d$ & $a$ & $d$ & $d$ \\
\hline$b$ & $a$ & $b$ & $a$ & $d$ & $d$ \\
\hline$c$ & $a$ & $d$ & $c$ & $d$ & $e$ \\
\hline$d$ & $a$ & $d$ & $a$ & $d$ & $d$ \\
\hline$e$ & $a$ & $d$ & $c$ & $d$ & $e$ \\
\hline
\end{tabular}

Define an IFS $A=<x, \mu_{A}, \gamma_{A}>$ by

$$
\mu_{A}: S \rightarrow[0,1] \mid \mu_{A}(x)=\left\{\begin{array}{l}
0.60 \text { if } x=a, \\
0.40 \text { if } x=b, \\
0.30 \text { if } x=c, \\
0.60 \text { if } x=d, \\
0.20 \text { if } x=e,
\end{array}\right.
$$

and

$$
\gamma_{A}: S \rightarrow[0,1] \mid \gamma_{A}(x)=\left\{\begin{array}{l}
0.30 \text { if } x=a, \\
0.40 \text { if } x=b, \\
0.60 \text { if } x=c, \\
0.30 \text { if } x=d, \\
0.50 \text { if } x=e .
\end{array}\right.
$$

Then $A=<x, \mu_{A}, \gamma_{A}>$ is an $(\bar{\epsilon}, \bar{\epsilon} \vee \bar{q})$-intuitionistic fuzzy interior ideals of $S$ for all $t, t_{1}, t_{2} \in(0,1]$ and $s, s_{1}, s_{2} \in[0,1)$. 
An intuitionistic fuzzy subset $A=<x, \mu_{A}(x), \gamma_{A}(x)>$ of $S$ is an $(\bar{\epsilon}, \bar{\epsilon} \vee \bar{q})$ -intuitionistic fuzzy interior ideal of $S$ it and only if for all $x, y, a \in S$, the following hold.

( $C_{5}$ ). $\quad \max \left\{\mu_{A}(x y), 0.5\right\} \geq \min \left\{\mu_{A}(x), \mu_{A}(y)\right\}$

$\min \left\{\gamma_{A}(x y), 0.5\right\} \leq \max \left\{\gamma_{A}(x), \gamma_{A}(y)\right\}$,

$\left(C_{6}\right) . \quad \max \left\{\mu_{A}(x a y), 0.5\right\} \geq \mu_{A}(a)$ and $\min \left\{\gamma_{A}(x a y), 0.5\right\} \leq \gamma_{A}(a)$.

Proof

$$
\begin{aligned}
& \left(C_{3}\right) \Rightarrow\left(C_{5}\right) \text {. If } \\
& \qquad \begin{aligned}
\max \left\{\mu_{A}(a b), 0.5\right\} & <t=\min \left\{\mu_{A}(a), \mu_{A}(b)\right\} \\
& \text { and } \\
\min \left\{\gamma_{A}(a b), 0.5\right\}>s & =\max \left\{\gamma_{A}(a), \gamma_{A}(b)\right\}
\end{aligned}
\end{aligned}
$$

for some $a, b \in S$ and $t \in(0.5,1], \quad s \in[0,0.5)$. Then $[a b ;(t, s)] \bar{\epsilon} A$ but $[a ;(t, s)] \in A,[b ;(t, s)] \in A$, also $[a ;(t, s)] q A[b ;(t, s)] q A$, a contradiction by ( $\left.C_{3}\right)$.

$\left(C_{5}\right) \Rightarrow\left(C_{3}\right) . \quad$ If $\quad\left[a b ; \min \left\{t_{1}, t_{2}\right\}, \max \left\{s_{1}, s_{2}\right\}\right] \bar{\in} A$, then $\mu_{A}(a b)<\min \left\{t_{1}, t_{2}\right\}$ and $\gamma_{A}(a b)>\max \left\{s_{1}, s_{2}\right\}$. We consider the following two cases:

Case I: If

$$
\mu_{A}(a b) \geq \min \left\{\mu_{A}(a), \mu_{A}(b)\right\}
$$

\section{And}

$$
\gamma_{A}(a b) \leq \max \left\{\gamma_{A}(a), \gamma_{A}(b)\right\},
$$

then,

$$
\begin{gathered}
\min \left\{\mu_{A}(a), \mu_{A}(b)\right\}<\min \left\{t_{1}, t_{2}\right\} \\
\text { And } \\
\max \left\{\gamma_{A}(a), \gamma_{A}(b)\right\}>\max \left\{s_{1}, s_{2}\right\},
\end{gathered}
$$

it follows that $\mu_{A}(a)<t_{1}, \gamma_{A}(a)>s_{1}$ or $\mu_{A}(b)<t_{2}, \gamma_{A}(b)>s_{2}$. Consequently $\left[a ;\left(t_{1}, s_{1}\right)\right] \in A$ or $\left[b ;\left(t_{2}, s_{2}\right)\right] \bar{\epsilon} A$.

Case II: If

$$
\mu_{A}(a b)<\min \left\{\mu_{A}(a), \mu_{A}(b)\right\}
$$

$$
\text { And }
$$

$$
\gamma_{A}(a b)>\max \left\{\gamma_{A}(a), \gamma_{A}(b)\right\}
$$

then, by $\left(C_{5}\right)$ we have

$$
0.5 \geq \min \left\{\mu_{A}(a), \mu_{A}(b)\right\}, 0.5 \leq \max \left\{\gamma_{A}(a), \gamma_{A}(b)\right\} .
$$

Let $\left[a ;\left(t_{1}, s_{1}\right)\right] \in A$ or $\left[b ;\left(t_{2}, s_{2}\right)\right] \in A$, then,

$$
0.5 \geq \mu_{A}(a) \geq t_{1}, 0.5 \leq \gamma_{A}(a) \leq s_{1}
$$

Or

$$
0.5 \geq \mu_{A}(b) \geq t_{2}, 0.5 \leq \gamma_{A}(b) \leq s_{2} .
$$

It follows that $\left[a ;\left(t_{1}, s_{1}\right)\right] \bar{q} A$ or $\left[b ;\left(t_{2}, s_{2}\right)\right] \bar{q} A$. Thus, in both case we see that $\left[a ;\left(t_{1}, s_{1}\right)\right]^{\bar{\epsilon}} \vee \bar{q} A$ or $\left[b ;\left(t_{2}, s_{2}\right)\right] \bar{\in} \vee \bar{q} A$.

$$
\begin{aligned}
& \left(C_{4}\right) \Rightarrow\left(C_{6}\right) \text { Let us consider } \\
& \qquad \max \left\{\mu_{A}(x a y), 0.5\right\}<t=\mu_{A}(a),
\end{aligned}
$$

$$
\text { And }
$$

$\min \left\{\gamma_{A}(x a y), 0.5\right\}>s=\gamma_{A}(a)$, for some $x, y, a \in S$ and $t \in(0.5,1], s \in[0,0.5)$, then $[x a y ;(t, s)] \bar{\epsilon} A$ but $[a ;(t, s)] \in A$, also $[a ;(t, s)] q A$, a contradiction by $\left(C_{4}\right)$.

$\left(C_{6}\right) \Rightarrow\left(C_{4}\right)$. Let $[x a y ;(t, s)] \bar{\epsilon} A$, then $\mu_{A}(x a y)<t$ and $\gamma_{A}(x a y)>s$ for some $x, y, a \in S$. We consider the following two cases:

Case I: If $\mu_{A}(x a y) \geq \mu_{A}(a), \gamma_{A}(x a y) \leq \gamma_{A}(a)$, then $\mu_{A}(a)<t, \gamma_{A}(a)>s$, it follows that $[a ;(t, s)] \bar{\epsilon} A$.

Case II: If $\mu_{A}(x a y)<\mu_{A}(a), \gamma_{A}(x a y)>\gamma_{A}(a)$, then by $\left(C_{6}\right)$ we have $0.5 \geq \mu_{A}(a), \quad 0.5 \leq \gamma_{A}(a)$. Let $[a ;(t, s)] \in A$, then $0.5 \geq \mu_{A}(a) \geq t$, $0.5 \leq \gamma_{A}(a) \leq s$. It follows that $[a ;(t, s)] \bar{q} A$. Thus, in both case we see that $[a ;(t, s)] \bar{\epsilon} \vee \bar{q} A$.

\subsection{Definition}

An intuitionistic fuzzy subset $A=<x, \mu_{A}(x), \gamma_{A}(x)>$ of $S$ is called an $(\bar{\epsilon}, \bar{\epsilon})$-intuitionistic fuzzy interior ideal of $S$, if for all $x, y, a \in S$, $t, t_{1}, t_{2} \in(0,1]$ and $s, s_{1}, s_{2} \in[0,1)$, satisfies the following conditions:

$\left(C_{7}\right) . \quad\left[x y ; \min \left\{t_{1}, t_{2}\right\}, \max \left\{s_{1}, s_{2}\right\}\right] \bar{\epsilon} A \rightarrow\left[x ;\left(t_{1}, s_{1}\right)\right] \bar{\epsilon} A$

or $\left[y ;\left(t_{2}, s_{2}\right)\right] \bar{\epsilon} A$,

$\left(C_{8}\right) . \quad[x a y ;(t, s)] \bar{\epsilon} A \rightarrow[a ;(t, s)] \bar{\epsilon}$.

\section{$3.5 \quad$ Example}

Consider a semigroup $S=\{0, a, b, c\}$ with the following multiplication table:

\begin{tabular}{|c|c|c|c|c|}
\hline. & 0 & $a$ & $b$ & $c$ \\
\hline 0 & 0 & 0 & 0 & 0 \\
\hline$a$ & 0 & 0 & 0 & 0 \\
\hline$b$ & 0 & 0 & 0 & $a$ \\
\hline$c$ & 0 & 0 & $a$ & $b$ \\
\hline
\end{tabular}

Define an IFS $A=<x, \mu_{A}, \gamma_{A}>$ by

$$
\mu_{A}: S \rightarrow[0,1] \mid \mu_{A}(x)=\left\{\begin{array}{l}
0.30 \text { if } x=0, \\
0.20 \text { if } x=a, \\
0.40 \text { if } x=b, \\
0.20 \text { if } x=c,
\end{array}\right.
$$

And

$$
\gamma_{A}: S \rightarrow[0,1] \mid \gamma_{A}(x)=\left\{\begin{array}{l}
0.50 \text { if } x=0, \\
0.60 \text { if } x=a, \\
0.30 \text { if } x=b, \\
0.40 \text { if } x=c .
\end{array}\right.
$$

Then $A=<x, \mu_{A}, \gamma_{A}>$ is an $(\bar{\epsilon}, \bar{\epsilon})$-intuitionistic fuzzy interior ideals of $S$ for all $t, t_{1}, t_{2} \in(0,1]$ and $s, s_{1}, s_{2} \in[0,1)$.

\subsection{Theorem}

Let $A=<x, \mu_{A}(x), \gamma_{A}(x)>$ be an intuitionistic fuzzy subset of $S$. Then $A$ is an intuitionistic fuzzy interior ideal of $S$ if $A$ is an $(\bar{\epsilon}, \bar{\epsilon})$-intuitionistic fuzzy interior ideal of $S$.

\section{Proof}

Let $A$ is an intuitionistic fuzzy interior ideal of $S$ and $\left[x y ; \min \left\{t_{1}, t_{2}\right\}, \max \left\{s_{1}, s_{2}\right\}\right] \in A, \quad$ then $\quad \mu_{A}(x y)<\min \left\{t_{1}, t_{2}\right\}$, $\gamma_{A}(x y)>\max \left\{s_{1}, s_{2}\right\}$. By $\left(C_{1}\right)$ we see that

$$
\min \left\{\mu_{A}(x), \mu_{A}(y)\right\} \leq \mu_{A}(x y)<\min \left\{t_{1}, t_{2}\right\},
$$


$\max \left\{\gamma_{A}(x), \gamma_{A}(y)\right\} \geq \gamma_{A}(x y)>\max \left\{s_{1}, s_{2}\right\}$.

Follows that $\left[x ; \min \left\{t_{1}, t_{2}\right\}, \max \left\{s_{1}, s_{2}\right\}\right] \bar{\epsilon} A$ or

$\left[y ; \min \left\{t_{1}, t_{2}\right\}, \max \left\{s_{1}, s_{2}\right\}\right] \bar{\epsilon} A$.

Finally, if $[x a y ;(t, s)] \bar{\epsilon}$, then $\mu_{A}(x a y)<t$ and $\gamma_{A}(x a y)>s$ and by $\left(C_{2}\right.$ ) we have

$$
\begin{gathered}
\mu_{A}(a) \leq \mu_{A}(x a y)<t, \\
\text { And } \\
\gamma_{A}(a) \geq \gamma_{A}(x a y)>s .
\end{gathered}
$$

This shows that $[a ;(t, s)] \bar{\epsilon} A$.

From the Theorem (3.6) it is clear that every intuitionistic fuzzy interior ideal is an $(\bar{\epsilon}, \bar{\epsilon})$-intuitionistic fuzzy interior ideal and it is obvious that every $(\bar{\epsilon}, \bar{\epsilon})$-intuitionistic fuzzy interior ideal of $S$ is an $(\bar{\epsilon}, \bar{\epsilon} \vee \bar{q})$ intuitionistic fuzzy interior ideal of $S$.

In the next theorem, we give a condition for an $(\bar{\epsilon}, \bar{\epsilon} \vee \bar{q})$-intuitionistic fuzzy interior ideal of $S$ to be an $(\bar{\epsilon}, \bar{\epsilon})$-intuitionistic fuzzy interior ideal of $S$.

\subsection{Theorem}

Let $A=\left\langle x, \mu_{A}, \gamma_{A}\right\rangle$ be an $(\bar{\epsilon}, \bar{\epsilon} \vee \bar{q})$-intuitionistic fuzzy interior ideal of $S$. If $\mu_{A}(x)>0.5$ and $\gamma_{A}(x)<0.5$ for all $x \in S$, then $A=\left\langle x, \mu_{A}, \gamma_{A}\right\rangle$ is an $(\bar{\epsilon}, \bar{\epsilon})$-intuitionistic fuzzy interior ideal of $S$.

\section{Proof}

Let $A=\left\langle x, \mu_{A}, \gamma_{A}\right\rangle$ be an $(\bar{\epsilon}, \bar{\epsilon} \vee \bar{q})$-intuitionistic fuzzy interior ideal of $S$ and $\mu_{A}(x)>0.5$ and $\gamma_{A}(x)<0.5$ for all $x \in S$. Let $t_{1}, t_{2} \in(0,1]$, $s_{1}, s_{2} \in[0,1)$ and consider $\left[x y ; \min \left\{t_{1}, t_{2}\right\}, \max \left\{s_{1}, s_{2}\right\}\right] \bar{\in} A$ then $\mu_{A}(x y)<\min \left\{t_{1}, t_{2}\right\}, \gamma_{A}(x y)>\max \left\{s_{1}, s_{2}\right\}$. By $\left(C_{5}\right)$ we have

$$
\begin{aligned}
\min \left\{\mu_{A}(x), \mu_{A}(y)\right\} & \leq \max \left\{\mu_{A}(x y), 0.5\right\}<\max \left\{\min \left\{t_{1}, t_{2}\right\}, 0.5\right\} \\
& = \begin{cases}\min \left\{t_{1}, t_{2}\right\} & \text { if } \min \left\{t_{1}, t_{2}\right\} \geq 0.5, \\
0.5 & \text { if } \min \left\{t_{1}, t_{2}\right\}<0.5\end{cases}
\end{aligned}
$$

$\max \left\{\gamma_{A}(x), \gamma_{A}(y)\right\} \geq \min \left\{\gamma_{A}(x y), 0.5\right\}>\min \left\{\max \left\{s_{1}, s_{2}\right\}, 0.5\right\}$,

$$
=\left\{\begin{array}{ll}
0.5 & \text { if } \max \left\{s_{1}, s_{2}\right\} \geq 0.5, \\
\max \left\{s_{1}, s_{2}\right\} & \text { if } \max \left\{s_{1}, s_{2}\right\}<0.5 .
\end{array} \quad\right. \text { This }
$$

implies $\mu_{A}(x)<\min \left\{t_{1}, t_{2}\right\}, \gamma_{A}(x)>\max \left\{s_{1}, s_{2}\right\}$ or

$$
\mu_{A}(y)<\min \left\{t_{1}, t_{2}\right\}, \gamma_{A}(y)>\max \left\{s_{1}, s_{2}\right\} \text { i.e., }
$$$$
\left[x ;\left(\min \left\{t_{1}, t_{2}\right\}, \max \left\{s_{1}, s_{2}\right\}\right)\right] \bar{\epsilon} A,
$$

$$
\text { Or }
$$

$\left[y ;\left(\min \left\{t_{1}, t_{2}\right\}, \max \left\{s_{1}, s_{2}\right\}\right] \bar{\in} A\right.$.

Let $x, y, a \in S$ with $[x a y ;(t, s)] \bar{\epsilon} A$, then $\mu_{A}(x a y)<t, \gamma_{A}(x a y)>s$ and from $\left(C_{6}\right.$ ) we see that;

$$
\begin{aligned}
\mu_{A}(a) & \leq \max \left\{\mu_{A}(\text { xay }), 0.5\right\}<\max \{t, 0.5\}, \\
& =\left\{\begin{array}{l}
t \quad \text { if } t \geq 0.5, \\
0.5 \text { if } t<0.5,
\end{array}\right. \\
\gamma_{A}(a) & \geq \min \left\{\gamma_{A}(\text { xay }), 0.5\right\}>\min \{s, 0.5\}, \\
& = \begin{cases}s & \text { if } s \leq 0.5, \\
0.5 \text { if } s>0.5 .\end{cases}
\end{aligned}
$$

Follows that $[a ;(t, s)] \bar{\epsilon} A$. Consequently, $A=\left\langle x, \mu_{A}, \gamma_{A}\right\rangle$ is an $(\bar{\epsilon}, \bar{\epsilon})$ intuitionistic fuzzy interior ideal of $S$.

\section{Theorem}

For an intuitionistic fuzzy subset $A=\left\langle x, \mu_{A}, \gamma_{A}\right\rangle$ of $S$, the following are equivalent for all $t \in(0.5,1]$ and $s \in[0,0.5)$ :

$\left(C_{9}\right) . \quad A$ is an $(\bar{\epsilon}, \bar{\epsilon} \vee \bar{q})$-intuitionistic fuzzy interior ideal of $S$.

$\left(C_{10}\right) . \quad C_{(t, s)}(A)(\neq \phi)$ is an interior ideal of $S$.

\section{Proof}

Assume that $A$ is an $(\bar{\epsilon}, \bar{\epsilon} \vee \bar{q})$-intuitionistic fuzzy interior ideal of $S$ and $C_{(t, s)}(A) \neq \phi$. Let $x, y \in C_{(t, s)}(A)$, then $\mu_{A}(x) \geq t, \gamma_{A}(x) \leq s$ and $\mu_{A}(y) \geq t$, $\gamma_{A}(y) \leq s \cdot\left(C_{5}\right)$ implies that

$$
\max \left\{\mu_{A}(x y), 0.5\right\} \geq \min \left\{\mu_{A}(x), \mu_{A}(y)\right\} \geq \min \{t, t\}=t,
$$

\section{And}

$\min \left\{\gamma_{A}(x y), 0.5\right\} \leq \max \left\{\gamma_{A}(x), \gamma_{A}(y)\right\} \leq \max \{s, s\}=s$. Thus, $x y \in C_{(t, s)}(A)$.

Next, we suppose $x, y, a \in S$ with $a \in C_{(t, s)}(A)$, then $\mu_{A}(a) \geq t, \gamma_{A}(a) \leq s$. From $\left(C_{6}\right)$, we have

$$
\max \left\{\mu_{A}(x a y), 0.5\right\} \geq \mu_{A}(a) \geq t,
$$

\section{And}

$$
\min \left\{\gamma_{A}(x a y), 0.5\right\} \leq \gamma_{A}(a) \leq s
$$

Thus $x a y \in C_{(t, s)}(A)$. Consequently $C_{(t, s)}(A)$ is an interior ideal of $S$.

Conversely, let $C_{(t, s)}(A)$ is an interior ideal of $S$. Assume that there exist $a, b \in S$ such that

$$
\begin{gathered}
\max \left\{\mu_{A}(a b), 0.5\right\}<\min \left\{\mu_{A}(a), \mu_{A}(b)\right\}, \\
\text { And }
\end{gathered}
$$$$
\min \left\{\gamma_{A}(a b), 0.5\right\}>\max \left\{\gamma_{A}(a), \gamma_{A}(b)\right\},
$$

then,

$$
\max \left\{\mu_{A}(a b), 0.5\right\}<t_{0} \leq \min \left\{\mu_{A}(a), \mu_{A}(b)\right\},
$$

\section{And}

$$
\min \left\{\gamma_{A}(a b), 0.5\right\}>s_{0} \geq \max \left\{\gamma_{A}(a), \gamma_{A}(b)\right\},
$$

for some $t_{0} \in(0.5,1]$ and $s_{0} \in[0,0.5)$, it follows that $a \in C_{\left(t_{0}, s_{0}\right)}(A)$ and $b \in C_{\left(t_{0}, s_{0}\right)}(A)$ but $a b \bar{\in} C_{\left(t_{0}, s_{0}\right)}(A)$, a contradiction.

Therefore,

$$
\max \left\{\mu_{A}(a b), 0.5\right\} \geq \min \left\{\mu_{A}(a), \mu_{A}(b)\right\},
$$

\section{And}

$$
\min \left\{\gamma_{A}(a b), 0.5\right\} \leq \max \left\{\gamma_{A}(a), \gamma_{A}(b)\right\},
$$

for all $x, y \in S$.

Next we consider,

$\max \left\{\mu_{A}(x a y), 0.5\right\}<\mu_{A}(a)$ and $\min \left\{\gamma_{A}(x a y), 0.5\right\}>\gamma_{A}(a)$ for some $x, y, a \in S$, then there exist $t_{1} \in(0.5,1]$ and $s_{1} \in[0,0.5)$ such that $\max \left\{\mu_{A}(x a y), 0.5\right\}<t_{1} \leq \mu_{A}(a)$ and $\min \left\{\gamma_{A}(x a y), 0.5\right\}>s_{1} \geq \gamma_{A}(a)$, it follows that $x a y \bar{\in} C_{\left(t_{1}, s_{1}\right)}(A)$ but $a \in C_{\left(t_{1}, s_{1}\right)}(A)$, a contradiction. Therefore, $\max \left\{\mu_{A}(x a y), 0.5\right\} \geq \mu_{A}(a)$ and $\min \left\{\gamma_{A}(x a y), 0.5\right\} \leq \gamma_{A}(a)$ for all $x, y, a \in S$. Consequently, $A$ is an $(\bar{\epsilon}, \bar{\epsilon} \vee \bar{q})$-intuitionistic fuzzy interior ideal of $S$.

For any IFS $A=\left\langle x, \mu_{A}, \gamma_{A}\right\rangle$ of $S, t \in(0,1]$ and $s \in[0,1)$, we consider two 
subsets:

$$
\begin{aligned}
Q_{(t, s)}(A) & :=\{x \in S \mid[x ;(t, s)] q A\}, \\
{[A]_{(t, s)} } & :=\{x \in S \mid[x ;(t, s)] \in \vee q A\} .
\end{aligned}
$$

It is obvious that $[A]_{(t, s)}=C_{(t, s)}(A) \cup Q_{(t, s)}(A)$.

\subsection{Proposition}

Let $A=\left\langle x, \mu_{A}, \gamma_{A}\right\rangle$ is an $(\bar{\epsilon}, \bar{\epsilon} \vee \bar{q})$-intuitionistic fuzzy interior ideal of $S$, then $Q_{(t, s)}(A)(\neq \phi)$ is an interior ideal of $S$ for all $t \in(0.5,1], s \in[0,0.5)$.

\section{Proof}

Consider $A=\left\langle x, \mu_{A}, \gamma_{A}\right\rangle$ is an $(\bar{\epsilon}, \bar{\epsilon} \vee \bar{q})$-intuitionistic fuzzy interior ideal of $S$. Let $t \in(0.5,1]$ and $s \in[0,0.5)$ such that $Q_{(t, s)}(A) \neq \phi$. Let $x, y \in Q_{(t, s)}(A)$ then $\mu_{A}(x)+t>1, \quad \gamma_{A}(x)+s<1 \quad$ and $\quad \mu_{A}(y)+t>1$, $\gamma_{A}(y)+s<1 .\left(C_{5}\right)$ implies that

$$
\begin{aligned}
\max \left\{\mu_{A}(x y), 0.5\right\} & \geq \min \left\{\mu_{A}(x), \mu_{A}(y)\right\} \\
& >\min \{1-t, 1-t\}=1-t, \\
& \text { And } \\
\min \left\{\gamma_{A}(x y), 0.5\right\} & \leq \max \left\{\gamma_{A}(x), \gamma_{A}(y)\right\} \\
& <\max \{1-s, 1-s\}=1-s .
\end{aligned}
$$

Thus $x y \in Q_{(t, s)}(A)$.

Next we suppose $x, y, a \in S$ such that $a \in Q_{(t, s)}(A)$, then $\mu_{A}(a)+t>1$, $\gamma_{A}(a)+s<1 \cdot\left(C_{6}\right)$, implies that

$$
\max \left\{\mu_{A}(x a y), 0.5\right\} \geq \mu_{A}(a)>1-t,
$$

And

$$
\min \left\{\gamma_{A}(x a y), 0.5\right\} \leq \gamma_{A}(a)<1-s .
$$

Follows that $Q_{(t, s)}(A)$ is an interior ideal of $S$.

\section{CONCLUSION}

In the world of contemporary mathematics, the use of algebraic structures in computer science, control theory and fuzzy automata theory always gain the interest of researchers. Algebraic structures particularly semigroups play a key role in such applied branches. Further, the fuzzification of several subsystems of semigroups are used in various models involving uncertainties. In this paper, we introduced new types of subsystems of semigroup called $(\bar{\epsilon}, \bar{\epsilon} \vee \bar{q})$-fuzzy subsystems and characterized semigroups in terms of $(\bar{\epsilon}, \bar{\epsilon} \vee \bar{q})$-intuitionistic fuzzy interior ideals and $(\bar{\epsilon}, \bar{\epsilon})$-intuitionistic fuzzy interior ideals. Finally, interior ideals and intuitionistic fuzzy interior ideal of type $(\bar{\epsilon}, \bar{\epsilon} \vee \bar{q})$ are connected by intuitionistic fuzzy level subset.

\section{REFERENCES}

[1] Zadeh, L. A. 1965. Fuzzy sets. Information and control, 8, 338-3353.

[2] Rosenfeld, A. 1971. Fuzzy groups. Journal of Mathematical Analysis and Applications, 35 (3), 512-517.

[3] Atanassov, K. 1986. Intuitionistic fuzzy Sets. Fuzzy Sets and Systems, 20 (1), 87-96.

[4] Biswas, R. 1989. Intuitionistic fuzzy subgroups. Mathematical Forum, $10,37-46$.
[5] Kim, K. H., and Jun, Y. B. 2001. Intuitionistic Fuzzy Interior Ideals of Semigroups. IJMMS, 275, 261-267.

[6] Yuan, X. H., Li, H. X., and Lee, E. S. 2010. On the definition of the intuitionistic fuzzy subgroups. Computers and Mathematics with Applications, 59, 3117-3129.

[7] Pu, P. M., and Liu, Y. M. 1980. Fuzzy topology I, neighborhood structure of a fuzzy point and Moore-Smith convergence. Journal of Mathematical Analysis and Applications, 76 (2), 571-599.

[8] Bhakat, S. K., and Das, P. 1996. $(\in, \in \vee q)$-fuzzy subgroups. Fuzzy Sets and Systems, 80, 359-368.

[9] Jun, Y. B., and Song, S. Z. 2006. Generalized fuzzy interior ideals in semigroups. Information Sciences, 176 (20), 3079-3093.

[10] Davvaz, B., and Khan, A. 2011. Characterizations of regular ordered semigroups in terms of-fuzzy generalized bi-ideals. Information Sciences, 181 (9), 1759-1770.

[11] Kazanci, O., and Yamak, S. 2008. Generalized fuzzy bi-ideals of semigroup. Soft Computing, 12 (11), 1119-1124.

[12] Shabir, M., Jun, Y. B., and Nawaz, Y. 2010. Characterization of regular semigroups by -fuzzy ideals. Computer and Mathematics with Applications, 60, 1473-1493.

[13] Shabir, M., Jun, Y. B., and Nawaz, Y. 2010. Semigroups characterized by -fuzzy ideals. Computer and Mathematics with Applications, 59, 161175 .

[14] Khan, A., Jun, Y., Abbasi, B., Z. 2012. Characterizations of ordered semigroups in terms of $(\in, \in \vee q)$-fuzzy interior ideals. Neural Computing and Applications, 21, 433-440.

[15] Zhan, J., and Jun, Y. B. 2010. Generalized fuzzy interior ideals of semigroups. Neural Computing and Applications, 19, 515-519.

[16] Shabir, M., Nawaz, Y., and Ali, M. 2011. Characterizations of Semigroups by $\left(\bar{\epsilon}, \bar{\in} \vee \overline{\mathrm{q}}_{k}\right)$-fuzzy Ideals. World Applied Sciences Journal, $14,(12), 1866-1878$.

[17] Davvaz, B., Kazanci, O., and Yamak, S. 2009. Generalized fuzzy n-ary subpolygroups endowed with interval valued membership functions, Journal Intelligent and Fuzzy Systems, 20 (4-5), 159-168.

[18] Davvaz, B., and Mozafar, Z. 2009. -fuzzy Lie subalgebra and ideals. International Journal of Fuzzy Systems, 11 (2), 123-129.

[19] Davvaz, B., and Corsini, P. 2008. On $(\alpha, \beta)$-fuzzy $H_{v}$-ideals of $H_{v}$ rings. Iranian Journal of Fuzzy System, 5 (2), 35-47.

[20] Ma, X., Zhan, J., and Jun, Y. B. 2008. Interval valued $(\in, \in \vee q)$-fuzzy ideals of pseudo-MV algebras. International Journal of Fuzzy Systems, 10 (2), 84-91.

[21] Mordeson, J. N., Malik, D. S., and Kuroki, N. 2003. Fuzzy Semigroups, Studies in Fuzziness and Soft Computing, Springer-Verlag, Berlin, 131.

[22] Yin, Y., and Zhan, J. 2010. New types of fuzzy filters of $B L$-algebras. Computer Mathematics with Applications, 60, 2115-2125.

[23] Yuan, X., Zhang, C., and Ren, Y. 2003. Generalized fuzzy groups and many-valued implications. Fuzzy Sets and Systems, 138, 205-211.

[24] Zhan, J., Davvaz, B., Shum, K. P. 2009. A new view of fuzzy hyper quasigroups. Journal of Intelligent and Fuzzy Systems, 20, 147-157. 